\title{
COEFFICIENT MULTIPLIERS OF BLOCH FUNCTIONS
}

BY

\section{J. M. ANDERSON AND A. L. SHIELDS}

ABSTRACT. The class $B$ of Bloch functions is the class of all those analytic functions in the open unit disc for which the maximum modulus is bounded by $c /(1-r)$ on $|z|<r$. We study the absolute values of the Taylor coefficients of such functions. In particular, we find all coefficient multipliers from $l^{p}$ into $B$ and from $B$ into $l^{p}$. We find the second Köthe dual of $B$ and show its relevance to the multiplier problem. We identify all power series $\Sigma a_{n} z^{n}$ such that $\Sigma w_{n} a_{n} z^{n}$ is a Bloch function for every choice of the bounded sequence $\left\{w_{n}\right\}$. Analogous problems for $H^{p}$ spaces are discussed briefly.

1. Introduction. The class of functions $f$, analytic for $|z|<1$ with $f(0)=0$, for which

$$
\|f\|=\sup _{0<r<1}(1-r)\left|f^{\prime}(z)\right|<\infty \quad(r=|z|)
$$

will be denoted by $B$. These functions (without the restriction $f(0)=0$ ) are called the Bloch functions. One easily verifies that $B$ is complete in this norm, and is therefore a Banach space. We shall denote by $B_{0}$ the subspace consisting of those functions $f \in B$ for which

$$
\lim (1-r)\left|f^{\prime}(z)\right|=0 \quad\left(r \rightarrow 1^{-}\right) .
$$

It has been shown in [1] that $B$ is the second dual of $B_{0}$, the first dual being the space $A(1,1)$ of functions $g$, analytic for $|z|<1$ with $g(0)=0$, for which

$$
\|g\|=\int_{0}^{1} \int_{0}^{2 \pi}\left|g^{\prime}\left(r e^{i \phi}\right)\right| r d r d \phi<\infty .
$$

The pairing is given by $\langle f, g\rangle=\lim _{r \rightarrow 1}-\Sigma_{1}^{\infty} \hat{f}(n) \hat{g}(n) r^{n}$ where $f(z)=\Sigma \hat{f}(n) z^{n} \in B$ and $g(z)=\Sigma \hat{g}(n) z^{n} \in A(1,1)$.

It is well known that, with the usual notations, $H^{\infty} \subset B$. On the other hand, the Bloch function $\Sigma \mathbf{z}^{2^{n}}$ does not have bounded characteristic. Thus the space $B$ cuts across the usual function classes. Nonetheless some nice properties remain. For example, it is shown in [1] that the Bloch functions have radial 
limits on a dense set. In this paper we shall study the Taylor coefficients of Bloch functions. In particular we shall determine the coefficient multipliers of $B$ into $l^{p}$.

Given two vector spaces $A, B$ of sequences, we denote by $(A, B)$ the space of "multipliers" from $A$ to $B$. More precisely, $(A, B)=\left\{\lambda=\left\{\lambda_{n}\right\}:\left\{\lambda_{n} a_{n}\right\} \in\right.$ $B$ for every $\left.\left\{a_{n}\right\} \in A\right\}$. We note the following two trivial facts. Here $A, B, C$ are any three sequence spaces.

$$
\begin{gathered}
A \subset B \Rightarrow(B, C) \subset(A, C), \\
A \subset(B, C) \text { is equivalent to } B \subset(A, C) .
\end{gathered}
$$

We regard spaces of analytic functions, such as $B$ and $A(1,1)$ as being sequence spaces (Taylor coefficients). The above example of a gap series Bloch function shows that the sequence $\lambda_{n}=(\log (n+1))^{-1 / 2}(n \geqslant 1)$ does not belong to $\left(B, l^{2}\right)$. On the other hand, it follows from (1.1) by Parseval's formula that, for $f \in B$,

$$
\sum_{1}^{\infty} k^{2}|\hat{f}(k)|^{2} r^{2 k} \leqslant c /(1-r)^{2} .
$$

Fixing $n$, and choosing $r=1-2^{-n}$ one obtains (see the proof of Lemma 8 for more details)

$$
\sum_{1+2^{n}}^{2^{n+1}}|\hat{f}(k)|^{2} \leqslant c_{1} \quad(n=1,2, \ldots) .
$$

Thus for any $\alpha>1 / 2$ the sequence $\lambda_{n}=(\log (n+1))^{-\alpha}$ is in $\left(B, l^{2}\right)$.

2. Definitions and preliminary results. Let $I_{n}=\left\{k: 2^{n} \leqslant k<2^{n+1}\right\}$ $(n=0,1, \ldots)$.

Definition 1. For $1 \leqslant \alpha, \beta \leqslant \infty$ we denote by $l(\alpha, \beta)$ the set of those sequences $\left\{a_{k}\right\}(k \geqslant 1)$ for which

$$
\left\{\left(\sum_{I_{n}}\left|a_{k}\right|^{\alpha}\right)^{1 / \alpha}\right\}_{n=0}^{\infty} \in l^{\beta} \quad(\alpha<\infty)
$$

and

$$
\left\{\sup _{k \in I_{n}}\left|a_{k}\right|\right\}_{n=0}^{\infty} \in l^{\beta} \quad(\alpha=\infty)
$$

These spaces are Banach spaces under the obvious norms. See C. N. Kellogg [9] for further information on them. We remark that $l(p, p)=l^{p}$. 
In studying the coefficient multipliers of $B$ we will identify $\left(B, l^{p}\right)$ with the space of multipliers from a larger space into $l^{p}$. Let $E$ denote the space of functions $f$, analytic in $|z|<1$ with $f(0)=0$, for which

$$
\|f\|_{E}=\sup _{0<r<1}(1-r)\left\{\int_{0}^{2 \pi}\left|f^{\prime}\left(r e^{i \theta}\right)\right|^{2} d \theta\right\}^{1 / 2}<\infty .
$$

We shall show that $\left(B, l^{p}\right)=\left(E, l^{p}\right)$. We require some general results on duality of sequences spaces and on multipliers into spaces such as $l^{p}$.

Various concepts of duality for sequence spaces are given in [5], [6], and [10]. Let $D$ be a fixed sequence space. Then the " $D$-dual" of a sequence space $A$, denoted by $A^{D}$, is defined to be $(A, D)$, the multipliers from $A$ to $D$. The Köthe dual is obtained when $D=l^{1}$, and will be denoted $A^{K}$. The Abel dual, with which we shall mainly be concerned, is obtained when $D$ is the space of Abel-summable sequences, that is, the space of sequences $\left\{d_{n}\right\}$ for which

$$
\lim \sum_{1}^{\infty} d_{n} r^{n} \quad\left(r \rightarrow 1^{-}\right)
$$

exists. We denote the Abel dual of $A$ by $A^{a}$. Note that when $d_{n} \geqslant 0$, then the existence of this limit is equivalent to $\Sigma d_{n}<\infty$. Clearly, $A^{K} \subset A^{a}$.

We have the following universal relations, valid for any $D$. Here $A^{D D}$ denotes $\left(A^{D}\right)^{D}$.

$$
\left\{\begin{array}{l}
\text { (a) } A \subset B \Rightarrow B^{D} \subset A^{D} \text {. } \\
\text { (b) } A \subset A^{D D} . \\
\text { (c) } A^{D}=A^{D D D} .
\end{array}\right.
$$

In general, $A \neq A^{D D}$. For example, in either Abel or Köthe duality if $A=c_{0}$ (sequences tending to 0 ), then $A^{D}=l^{1}$ and $A^{D D}=l^{\infty}$.

LEMMA 1. (i) $(A, B) \subset\left(B^{D}, A^{D}\right)$.

(ii) $\left(B^{D}, A^{D}\right)=\left(A^{D D}, B^{D D}\right)$.

Proof. (i) Let $\left\{\lambda_{n}\right\} \in(A, B)$, and $\left\{\mu_{n}\right\} \in B^{D}$. We must show that $\left\{\lambda_{n} \mu_{n}\right\} \in A^{D}$, in other words, that $\left\{\lambda_{n} \mu_{n} a_{n}\right\} \in D$ for all $\left\{a_{n}\right\} \in A$. But $\left\{\lambda_{n} \mu_{n} a_{n}\right\}=\left\{\left(\lambda_{n} a_{n}\right) \mu_{n}\right\} \in D$ since $\left\{\lambda_{n} a_{n}\right\} \in B$.

(ii) By (i) and (c) we have

$$
\left(B^{D}, A^{D}\right) \subset\left(A^{D D}, B^{D D}\right) \subset\left(B^{D D D}, A^{D D D}\right)=\left(B^{D}, A^{D}\right) .
$$

Note that equality need not hold in (i), even for Köthe or Abel duality. Let $A=c$, the convergent sequences. Then $A^{D}=l^{1}$ for Köthe or Abel duality. Then $(A, A)=c$ (since $c$ contains the constant sequence 1$)$, but $\left(A^{D}, A^{D}\right)=$ $\left(l^{1}, l^{1}\right)=l^{\infty}$. 
A sequence space $A$ is said to be solid if, whenever it contains $\left\{a_{n}\right\}$ it also contains all sequences $\left\{b_{n}\right\}$ with $\left|b_{n}\right| \leqslant\left|a_{n}\right|$. Such spaces are also called normal ([10, $\$ 30$, p. 405$])$. Equivalently, $A$ is solid if $l^{\infty} \subset(A, A)$. Note that for solid spaces the Abel dual and the Köthe dual coincide: $A^{K}=A^{a}$.

LEMMA 2. If $A$ is a sequence space then there is a largest solid subspace, $s(A)$, contained within it, and a smallest solid superspace, $S(A)$, containing it. Furthermore, $s(A)=\left(l^{\infty}, A\right)$.

Proof. The space $S(A)$ is the intersection of all the solid spaces that contain $A$ (for example, the space of all sequences).

Let $s(A)=\left(l^{\infty}, A\right)$. It is trivial to verify that $s(A)$ is a solid subspace of $A$. Now let $B \subset A$ be any other solid subspace. If $\left\{b_{n}\right\} \in B$ and $\left\{w_{n}\right\} \in l^{\infty}$ then $\left\{w_{n} b_{n}\right\} \in B \subset A$. Hence $\left\{b_{n}\right\} \in\left(l^{\infty}, A\right)$, and so $B \subset s(A)$.

Lemma 3. Let $X$ be any solid space. Then

(i) $(A, X)=(S(A), X)$,

(ii) $(X, A)=(X, s(A))$.

Proof. (i) Let $B=(A, X)$. Then $A \subset(B, X)$ by (1.3). But this is a solid space containing $A$ and so $S(A) \subset(B, X)$. Using (1.3) and (1.2) we have

$$
B \subset(S(A), X) \subset(A, X)=B .
$$

(ii) Let $B=(X, A)$, and let $\left(b_{n}\right) \in B,\left(x_{n}\right) \in X$. By Lemma $2, s(A)=$ $\left(l^{\infty}, A\right)$. Thus to show that $\left(b_{n} x_{n}\right) \in s(A)$ we must show that $\left(b_{n} x_{n} w_{n}\right)=$ $\left(b_{n}\left(x_{n} w_{n}\right)\right)$ is in $A$ for all $\left(w_{n}\right) \in l^{\infty}$, and this is obvious.

We now show that $s\left(A^{a}\right)=A^{K}$, the Köthe dual of $A$ (recall that $A^{K}=$ $\left.\left(A, l^{1}\right)\right)$.

LEMMA 4. (i) $s\left(A^{a}\right)=A^{K}$.

(ii) If $A^{a a}=A$, then $s(A)=\left(A^{a}\right)^{K}$.

(iii) If $X$ is solid and $X^{K K}=X$, then $(A, X)=\left(A^{K K}, X\right)$.

Proof. (i) From Lemmas 2 and 1 (ii) we have

$$
s\left(A^{a}\right)=\left(l^{\infty}, A^{a}\right)=\left(A^{a a}, l^{1}\right) \subset\left(A, l^{1}\right)=A^{K} .
$$

Conversely, $A^{K}$ is solid and $A^{K} \subset A^{a}$, so $A^{K} \subset s\left(A^{a}\right)$.

(ii) Same proof as in (i).

(iii) Since $s\left(A^{a}\right) \subset A^{a}$ we have $A \subset s\left(A^{a}\right)^{a}$. Hence from Lemmas 1 and 2, and from (1.2), we have

$$
(A, X) \subset\left(X^{K}, A^{K}\right) \subset\left(A^{K K}, X\right) \subset(A, X) .
$$


Note the significance of part (iii). Trivially, we have $A \subset A^{K K}$, and $A^{K K}$ is solid, so that $S(A) \subset A^{K K}$. In general, they do not coincide (eg. $A=c_{0}$; see also $\S 4$, Example 1). However, from an operational point of view (more precisely, when considering multipliers into any solid space $X$ that is "Köthe reflexive": $X^{K K}=X$ ) these two spaces are interchangeable. Since it is frequently very difficult to determine $S(A)$ precisely, we may use $A^{K K}$ as a reasonable substitute. Furthermore, it can be obtained from the relation $A^{K K}=s\left(A^{a}\right)^{a}$ whenever $s\left(A^{a}\right)$ has been obtained (and there are methods for finding $s\left(A^{a}\right)$ in many cases). Spaces for which $X^{K K}=X$ are sometimes called perfect $([10, \S 30$, p. 406]).

THEOREM. Let $X$ be any solid space with $X^{K K}=X$. Then

$$
(B, X)=(l(2, \infty), X) \text {. }
$$

The multipliers $\left(l(2, \infty), l^{p}\right)$ are easily determined, this has been done by Kellogg [8]. Thus we have the following result.

Corollary 1.

$$
\left(B, l^{p}\right)= \begin{cases}l\left(\frac{2 p}{2-p}, p\right), & 1 \leqslant p \leqslant 2, \\ l(\infty, p), & 2 \leqslant p \leqslant \infty .\end{cases}
$$

Since $B^{K}=\left(B, l^{1}\right)=l(2,1)$, we have identified the second Köthe dual of B.

Corollary 2. $B^{K K}=l(2, \infty)$.

3. Proof of Theorem 1. We shall use the Rademacher functions $\left\{r_{n}(t)\right\}$ (see [10, Chapter $\mathrm{V}, \S 8]$ for the elementary properties of these functions). In particular we require Khintchin's inequality [12, Chapter V, Theorem 8.4] which we state as follows.

LEMMA 5. Let $f(z)=\Sigma \hat{f}(n) z^{n}$ be analytic for $|z|<1$. Let

$$
f_{t}(z)=\sum r_{n}(t) \hat{f}(n) z^{n} \quad(0 \leqslant t \leqslant 1) .
$$

Then there are absolute constants $c_{1}$ and $c_{2}$ such that

$$
c_{1} \int_{0}^{1}\left|f_{t}(z)\right| d t \leqslant\left(\sum|\hat{f}(n)|^{2}|z|^{2 n}\right)^{1 / 2} \leqslant c_{2} \int_{0}^{1}\left|f_{t}(z)\right| d t, \quad|z|<1
$$


Definition 2. For $1 \leqslant \alpha, \beta<\infty$ we denote by $A(\alpha, \beta)$ the set of those functions $h(z)$ analytic for $|z|<1$ with $h(0)=0$, for which

$$
\int_{0}^{1}\left(\int_{0}^{2 \pi}\left|h^{\prime}\left(r e^{i \theta}\right)\right|^{\alpha} d \theta\right)^{\beta / \alpha} r d r<\infty .
$$

The corresponding spaces when $\alpha$ or $\beta=\infty$ are defined analogously. Note that $A(2, \beta)$ is a solid space $(1 \leqslant \beta \leqslant \infty)$ since the $d \theta$ integral may be replaced by a sum of squares of coefficients, by Parseval's formula. It is readily seen that these "mixed norm" function spaces are, in fact, Banach spaces under the obvious norms.

It was shown in [1] that $A(1,1)^{a}=B$, and that $B_{0}^{a}=A(1,1)$. It follows from (2.3) (c) that $B^{a}=A(1,1)$. Let $X$ be a solid space with $X^{a a}=X$. From Lemmas 1 (ii) and 3 (ii) we have

$$
(B, X)=\left(X^{a}, A(1,1)\right)=\left(X^{a}, s(A(1,1))\right) .
$$

We now identify $s(A(1,1))$, the largest solid subspace of $A(1,1)$.

LEMMA 6. The space $A(2,1)$ is the largest solid subspace of $A(1,1)$.

Proof. It is readily seen that $A(2,1)$ is a solid subspace of $A(1,1)$. To complete the proof we must show, by Lemma 2 , that $\left(l^{\infty}, A(1,1)\right) \subset A(2,1)$.

Let $\left\{a_{n}\right\} \in\left(l^{\infty}, A(1,1)\right)$. By the closed graph theorem, $\left\{a_{n}\right\}$ is a bounded linear transformation from $l^{\infty}$ into $A(1,1)$. In particular, if $f(z)=\Sigma a_{n} z^{n}$ and if $f_{t}$ is defined by (3.1), then there is a constant $c$ such that $\left\|f_{t}\right\| \leqslant c(0 \leqslant t \leqslant 1)$, where we are using the norm in $A(1,1)$. Thus

$$
\int_{0}^{1} \int_{0}^{2 \pi} \int_{0}^{1}\left|\sum r_{n}(t) n a_{n} z^{n-1}\right| s d s d \theta d t \leqslant c \quad\left(z=s e^{i \theta}\right) .
$$

Interchanging the order of integration and using Khintchin's inequality (Lemma 5) we have

$$
\int_{0}^{1}\left(\sum n^{2}\left|a_{n}\right|^{2} s^{2 n}\right)^{1 / 2} d s \leqslant c^{\prime}
$$

that is, $f \in A(2,1)$, which completes the proof.

Recall the space $E$ defined in (2.1).

LEMMA 7. $E=A(2,1)^{a}$.

Proof. At this time we prove only that $E \subset A(2,1)^{a}$. This will allow us to complete the proof of the theorem. Then we shall return and finish the proof of this lemma.

Let $f \in A(2,1), g \in E$ be given. Let $h_{1}(z)=(z f)^{\prime} / z$ (recall that $\left.f(0)=0\right)$, and let $h_{2}(z)=g^{\prime}(z)$. Then one verifies that, for $0<s<1$, 


$$
\sum_{1}^{\infty} \hat{f}(n) \overline{\hat{g}(n)} s^{2 n+2}=\frac{1}{\pi} \int_{0}^{s}\left(\int_{0}^{2 \pi} h_{1}(z) \overline{h_{2}(z)} d \theta\right)\left(s^{2}-r^{2}\right) r d r \quad\left(z=r e^{i \theta}\right)
$$

We must show that the limit exists as $s \rightarrow 1^{-}$. The integral on the right side of (3.4) may be rewritten as the integral over the open unit disc of the function: $h_{1}(z) \overline{h_{2}(z)} \phi_{s}(r)$, where $\phi_{s}(r)=\left(s^{2}-r^{2}\right) / \pi$ for $r<s$, and is 0 otherwise. This integrand is dominated by $\left|h_{1}(z) h_{2}(z)\left(1-r^{2}\right)\right|$ and so the existence of the limit will follow from the dominated convergence theorem once this last function is known to be integrable. We have

$$
\begin{aligned}
& \int_{0}^{1}\left(\int_{0}^{2 \pi}\left|h_{1}(z) h_{2}(z)\right| d \phi\right)(1-r) d r \\
& \quad<\int_{0}^{1}\left(\int_{0}^{2 \pi}\left|h_{1}\right|^{2} d \theta\right)^{1 / 2}\left(\int_{0}^{2 \pi}\left|h_{2}\right|^{2} d \theta\right)^{1 / 2}(1-r) d r .
\end{aligned}
$$

But by hypothesis $\left(\int\left|h_{1}\right|^{2} d \theta\right)^{1 / 2} \in L^{1}(d r)$, and $(1-r)\left(\int\left|h_{2}\right|^{2} d \theta\right)^{1 / 2}$ is bounded, and so the integral converges. This completes the proof of one half of the lemma. We postpone the proof of the other half.

Since $B \subset E$ we have, using (3.3) and the last two lemmas,

$$
(E, X) \subset(B, X)=\left(X^{a}, A(2,1)\right) \subset\left(A(2,1)^{a}, X\right) \subset(E, X) .
$$

Thus we have shown that $(B, X)=(E, X)$ for all solid spaces $X$ with $X^{K K}=X$. To complete the proof of the theorem we need to identify $E$ as a sequence space.

LEMMA 8. $E=l(2, \infty)$.

Proof. Let $f \in E$. From (2.1) and the Parseval relation we have

$$
\sum_{1}^{\infty} k^{2}|\hat{f}(k)|^{2} r^{2 k} \leqslant c\left(1 /\left(1-r^{2}\right)\right)^{2} \quad(0<r<1) .
$$

Put $r^{2}=1-N^{-1}$ and note that $r^{2 n}>c>0$ for $n \leqslant N$ ( $c$ will be used for a general constant, not necessarily the same at each occurrence). We have

$$
\sum_{1}^{N} k^{2}|\hat{f}(k)|^{2} \leqslant c N^{2} .
$$

Hence, with $I(n)=\left\{k: 2^{n} \leqslant k<2^{n+1}\right\}$ we have

$$
\sum_{I(n)}|\hat{f}(k)|^{2}<c
$$

Thus $f \in l(2, \infty)$.

Conversely, let $f \in l(2, \infty)$. Reversing the reasoning leads to (3.7), and we wish to show that this implies (3.5). Note that there is a constant $c$ such that 
$\Sigma_{m+1}^{2 m}|\hat{f}(k)|^{2} \leqslant c(m=1,2, \ldots)$. Indeed, let $m \in I(n)$. Then $(m, 2 m] \subset$ $I(n) \cup I(n+1)$, and the result follows. Hence if $N$ is given we have

$$
\sum_{1+j N}^{(j+1) N}|\hat{f}(n)|^{2} \leqslant c \quad(j=1,2, \ldots)
$$

since $(j N,(j+1) N) \subset(j N, 2 j N]$. Now let $r<1$ be given, and choose $N$ such that $1-(N-1)^{-1}<r^{2} \leqslant 1-N^{-1}$. Then

$$
\begin{aligned}
\sum_{1}^{\infty} k^{2}|\hat{f}(k)|^{2} r^{2 k} & \leqslant\left(\sum_{1}^{N}+\sum_{1+N}^{2 N}+\sum_{1+2 N}^{3 N}+\cdots\right) k^{2}|\hat{f}(k)|^{2}(1-1 / N)^{k} \\
& \leqslant c N^{2}+c(2 N)^{2}(1-1 / N)^{N}+c(3 N)^{2}(1-1 / N)^{2 N}+\cdots \\
& \leqslant c N^{2} \leqslant c /(1-r)^{2}
\end{aligned}
$$

(Let $\alpha=(1-1 / N)^{N}<e^{-1}$. Then $\Sigma k^{2} \alpha^{k-1}<\infty$.) The lemma is proved. This completes the proof of the theorem.

We now complete the proof of Lemma 7. From (3.5), if $X$ is any solid space for which $X^{a a}=X$, then

$$
(E, X)=(B, X)=\left(A(2,1)^{a}, X\right) .
$$

From Lemma 8 we have $E=l(2, \infty)$, a solid, Köthe-reflexive space (one verifies that $l(p, r)^{a}=l\left(p^{\prime}, r^{\prime}\right)$, where $p^{\prime}, r^{\prime}$ are the conjugate indices, and $\left.1 \leqslant p, r \leqslant \infty\right)$. Choose $X=l(2, \infty)$ in (3.9). Then $l^{\infty}=(l(2, \infty), l(2, \infty))=\left(A(2,1)^{a}, l(2, \infty)\right)$. Since the constant sequence 1 is in $l^{\infty}$, we see that $A(2,1)^{a} \subset l(2, \infty)=E$. This completes the proof of the lemma.

Using this result we can identify $A(2,1)$ as a sequence space.

LEMMA 9. $A(2,1)=l(2,1)$.

Proof. From Lemmas 7 and 8 we have $A(2,1)^{a}=l(2, \infty)$. Hence $A(2,1) \subset A(2,1)^{a a}=l(2,1)$.

Conversely, $B \subset E=l(2, \infty)$; hence $l(2,1) \subset B^{a}=A(1,1)$. Thus $l(2,1)$ is a solid subspace of $A(1,1)$ and hence lies in the greatest solid subspace, namely in $A(2,1)$. This completes the proof.

4. Multipliers and solid spaces. The previous discussion suggests a more wide-ranging investigation into solid subspaces and superspaces, and the question of when two spaces have the same multipliers into a given space. The basic problem is: given a sequence space $A$ to find $s(A)$ and $S(A)$, the largest solid subspace and the smallest solid superspace. As pointed out in Lemma 4 (iii) and the discussion following the proof, the second Köthe dual of $A, A^{K K}$, may 
be used as a substitute for $S(A)$ when $S(A)$ itself cannot be found. We now determine the largest solid subspace of $B$.

LEMMA 10. $l(1, \infty)$ is the largest solid subspace of $B$.

Proof. An argument similar to that in the proof of Lemma 8, where we proved the equivalence of (3.7) and (3.8), shows that $f$ is in $l(1, \infty)$ if and only if

$$
\sum_{1}^{N} k|f(k)| \leqslant c N \quad(\text { all } N) .
$$

Also, an argument similar to that used in going from (3.7) to (3.6) shows that, if (4.1) holds, then $f \in B$. Thus $l(1, \infty) \subset B$.

Finally, if $f \in B$ and if $\hat{f} \geqslant 0$, then an argument similar to the derivation of (3.8) from (3.6) shows that $f \in l(1, \infty)$. This completes the proof.

Thus we have

$$
l(1, \infty)=s(B) \subset B \subset S(B) \subset B^{K K}=l(2, \infty) .
$$

Presumably $S(B) \neq l(2, \infty)$. Similarly one can obtain

$$
l(2,1)=s(A(1,1)) \subset A(1,1) \subset S(A(1,1)) \subset A(1,1)^{K K}=l(\infty, 1) .
$$

We have used Theorem 3 of [4] which describes $A(1,1)^{K}$. Presumably $S(A(1,1)) \neq l(\infty, 1)$.

We now make a few remarks about the spaces $L^{p}$ (for the circle group, $|z|=1$ ). We shall consider these spaces as sequence spaces (Fourier coefficients), indexed from $-\infty$ to $\infty$. The Abel summable sequences $\left\{a_{n}\right\}$ are those for which

$$
\lim \sum_{-\infty}^{\infty} a_{n} r^{|n|} \text { exists }\left(r \rightarrow 1^{-}\right) \text {. }
$$

EXAMPLE 1. $L^{1}$. Here we have

$$
l^{2}=s\left(L^{1}\right) \subset L^{1} \subset S\left(L^{1}\right)=c_{0} .
$$

The first equality follows from [12, Chapter V, Theorem 8.12]. The last equality follows from the Riemann-Lebesgue lemma, and the fact that $L^{1} * c_{0}=$ $c_{0}$ (this notation means that we form all sequences $\left\{\hat{f}(n) w_{n}\right\}$ where $f \in L^{1}$ and $\left.\left\{w_{n}\right\} \in c_{0}\right)$. See, for example, [7]. A proof can also be based on [12, Chapter V, Theorem 1.5]. We deduce that $\left(L^{1}\right)^{K}=l^{1}$, and $\left(L^{1}\right)^{K K}=l^{\infty}$. Thus $S\left(L^{1}\right)$ $\neq\left(L^{1}\right)^{K K}$.

Example 2. $L^{p}(1<p \leqslant 2)$. Here we have

$$
l^{2}=s\left(L^{p}\right) \subset L^{p} \subset S\left(L^{p}\right) \subset l\left(p^{\prime}, 2\right) .
$$

Here $p^{\prime}$ denotes the conjugate index. The fact that $l^{2}=s\left(L^{p}\right)$ is a consequence of Example 1, since $L^{2} \subset L^{p} \subset L^{1}$. The fact that $L^{p} \subset l\left(p^{\prime}, 2\right)$ was shown by 
Kellogg [8]. Here neither $S\left(L^{p}\right)$ nor even $\left(L^{p}\right)^{K K}$ seem to be known.

EXAMPLE 3. $L^{p}(2<p<\infty)$. Here we have

$$
l\left(p^{\prime}, 2\right) \subset s\left(L^{p}\right) \subset L^{p} \subset S\left(L^{p}\right)=l^{2} .
$$

The first containment on the left follows by duality from Example 2. The fact that $S\left(L^{p}\right)=l^{2}$ is in [12, Chapter V, Theorem 8.16]. Here $s\left(L^{p}\right)$ does not seem to be known. It coincides with the set of unconditionally convergent Fourier series in $L^{p}$ (see [2], [3]).

EXAMPLE 4. $L^{\infty}$. We have

$$
l^{1}=s\left(L^{\infty}\right) \subset L^{\infty} \subset S\left(L^{\infty}\right) \subset\left(L^{\infty}\right)^{K K}=l^{2} .
$$

For the first equality note that, on the one hand, $l^{1}$ is a solid subspace of $L^{\infty}$, and that, on the other hand, if $f \subset L^{\infty}$ has positive Fourier coefficients, then $f \in l^{1}$ (consider the Fejér means of the Fourier series of $f$ ). The last equality follows from Example 1 and Lemma 4, and from the fact that $\left(L^{\infty}\right)^{a}=L^{1}$. This last was proved by Steinhaus (see [11, the converse to Parallelsatz 3, pp. 205-210]). A proof can also be based on [12, Chapter IV, Theorem 9.13]. Actually, one has $\left(L^{\infty}\right)^{K}=l^{2}$, from which the result above follows. Also, it seems to be the case that $S\left(L^{\infty}\right) \neq l^{2}$ (see [8, Chapter 7, §6, Exercise 2, p. 70]).

Our final example is the Hardy space $H^{1}$. It can be described as the subspace of $L^{1}$ consisting of those $f$ for which $\hat{f}(n)=0(n<0)$. Thus it may be viewed as a sequence space where the sequences are indexed from 0 to $\infty$. One could also consider the Hardy spaces $H^{p}(1<p<\infty)$ but the M. Riesz projection theorem ([12, Chapter VII, Theorem 2.4]) shows that the results here will be the same as for the corresponding spaces $L^{p}$.

EXAMPLE 5. $H^{1}$. We have

$$
l^{2}=s\left(H^{1}\right) \subset H^{1} \subset S\left(H^{1}\right) \subset c_{0} .
$$

Here $c_{0}$ is not the smallest solid subspace containing $H^{1}$, unlike the case of $L^{1}$. For example, a theorem of Hardy and Littlewood ([12, Chapter VII, Theorem 8.7]), says that

$$
\sum \frac{|f(n)|}{n+1} \leqslant \pi\|f\| \quad\left(f \in H^{1}\right) .
$$

Also, it follows from a result of Paley ([12, Chapter XII, Theorem 7.8]), that $H^{1} \subset l(\infty, 2)$. C. Fefferman (unpublished) has determined the Köthe dual of $H^{1}$. It consists of all those sequences $\left\{\lambda_{n}\right\}$ such that

$$
\sum_{k=1}^{\infty}\left(\sum_{j=k n+1}^{(k+1) n}\left|\lambda_{j}\right|\right)^{2} \leqslant c<\infty \quad(n=1,2, \ldots) .
$$




\section{BIBLIOGRAPHY}

1. J. M. Anderson, J. Clunie and C. Pommerenke, On Bloch functions and normal functions, J. Reine Angew. Math. 270 (1974), 12-37. MR 50 \#13536.

2. G. F. Bachelis, On the ideal of unconditionally convergent Fourier series in $L_{p}(G)$, Proc. Amer. Math. Soc. 27 (1971), 309-312. MR 42 \#6523.

3. - On the upper and lower majorant properties in $L^{p}(G)$, Quart. J. Math. Oxford Ser. 24 (1973), 119-128. MR 47 \#172.

4. P. L. Duren and A. L. Shields, Properties of $H^{p}(0<p<1)$ and its containing Banach space, Trans. Amer. Math. Soc. 141 (1969), 255-262. MR 39 \#6065.

5. D. J. H. Garling, The $\beta$ - and $\gamma$-duality of sequence spaces, Proc. Cambridge Philos. Soc. 63 (1967), 963-981. MR 36 \#1965.

6. - On symmetric sequence spaces, Proc. London Math. Soc. (3) 16 (1966), 85-106. MR 33 \#537.

7. E. Hewitt, The ranges of certain convolution operators, Math. Scand. 15 (1964), 147-155. MR 32 \#4471.

8. J. P. Kahane, Some random series of functions, Heath, Lexington, Mass., 1968. MR 40 \#8095.

9. C. N. Kellogg, An extension of the Hausdorff-Young theorem, Michigan Math. J. 18 (1971), 121-127. MR 43 \#6714.

10. G. Köthe, Topological vector spaces.I, Springer-Verlag, Berlin, 1960; English transl., Springer-Verlag, New York, 1969. MR 24 \#A411; 40 \#1750. 186-221.

11. H. Steinhaus, Additive and stetige Funktionaloperationen, Math. Z. 5 (1919),

12. A. Zygmund, Trigonometric series, 2nd rev. ed., Cambridge Univ. Press, New York, 1959. MR 21 \#6498.

\section{ENGLAND}

DEPARTMENT OF MATHEMATICS, UNIVERSITY COLLEGE, LONDON, w. C. 1,

DEPARTMENT OF MATHEMATICS, UNIVERSITY OF MICHIGAN, ANN ARBOR, MICHIGAN 48109 